\title{
SOME STATISTICAL PROPERTIES OF THE ROUGH SURFACE*
}

\author{
T. BALCERZAK \\ Solid State Physics Department, University of Łódź \\ Pomorska 149/153, 90-236 Eódź, Poland
}

\begin{abstract}
The temperature induced roughening of the solid surface is described by means of a discrete Gaussian model, using integral operator technique. On the basis of the exact statistical identities, the formulae for several local moments, i.e. $\left\langle h_{i}\right\rangle,\left\langle\left(h_{i}\right)^{2}\right\rangle$ and $\left\langle h_{i} h_{j}\right\rangle$ are derived. The results of computer calculations, including surface specific heat, are presented for three lattices with $z=3,4$, and 6 . In discussion, some further applications of the presented method are suggested.
\end{abstract}

PACS numbers: $05.50 .+q, 68.35 . \mathrm{Md}$

\section{Introduction}

The idea of surface roughening was developed originally by Burton and Cabrera $[1,2]$ in 1949 . They represented the crystal surface with vacancies and adatoms by a two-dimensional Ising model and studied the structure fluctuations at the Ising critical temperature.

The generalised model of Burton and Cabrera is commonly known as a solid-on-solid (SOS) model [3, 4]. At present, there is a variety of SOS models, one of the most important is a discrete Gaussian (DG) model introduced by Chui and Weeks [5, 6] in 1976.

A great deal of theoretical work has been done to clarify the existence and the nature of roughening phase transition within SOS models. Most of the results were obtained using renormalization group (RG) approach and Monte Carlo (MC) computer simulations. We will refer here to the review articles [6-10] summarising the main theoretical results.

The general conclusion from these papers is that, on a large scale, the height-to-height correlation function should diverge at some temperature and remains infinite above it. The phase transition is of continuous character and, in a contrast to the Ising models, does not exhibit any peak in the specific heat. As it has been suggested, this phase transition originates from the fact that the edge free energy of a step on a crystal facet becomes zero at some temperature. This, in turn, makes possible to move the steps freely, and the reference level on the

*This paper is supported by the grant $\mathrm{PB} / 1775 / 2 / 91$. 
facet is being lost. The crystal is called "rough" above the phase transition temperature and "smooth" below it. In particular, for DG model, the phase transition temperature is found to be $k T_{R} / J \approx 1.45$ for (001) face [6]. It has been shown that, in the most studied cases, the roughening phase transition is equivalent to the Kosterlitz-Thouless type [11].

The experimental observation of the roughening transition is difficult for several reasons. First of all, the crystals produced during growth are metastable and the relaxation time to obtain the equilibrium shape increases rapidly with the crystal size. This makes difficult to produce crystals of the equilibrium shape having sufficiently great size for observations [9]. For this reason, the experiments are usually carried out on the macroscopic metastable vicinal surfaces, on which the local equilibrium of the step configuration is achieved.

It turns out that for the most observable crystal facets the roughening transition temperature is out of physical range of the surface existence, i.e. is above the melting point. The lowering of the roughening transition temperature can be achieved when the high-index (vicinal) surfaces, which have relatively small atomic density, are used for observation [9].

Because of those difficulties, the experimental works concerning roughening have started only at eighties. Since then, many crystal surfaces have been studied. For instance, the ${ }^{4} \mathrm{He}$ solid-superliquid interface [12], the vicinal surfaces of $\mathrm{Cu}$ and Ni [13-16], Pt (111) surface [17], GaAs (001) and Si (001) surfaces [18], and recently, $\mathrm{Pb}(110), \mathrm{Au}(111)$ and $\mathrm{Au}(100)$ surfaces [19] have been investigated.

For those experiments many modern techniques have been involved, namely: grazing incidence X-ray scattering (GIXS) [13], helium beam diffraction [14, 15], thermal energy atom scattering (TEAS) [16], pulsed molecular beam epitaxy (MBE) [17], reflection high-energy electron diffraction (RHEED) [18], and X-ray photoelectron diffraction [19]. Another surface technique, low-energy electron diffraction (LEED), has been used for studying the growth and ordering of chemisorbed layers [20].

As is seen from this very short survey, the surface roughness has been intensively studied both theoretically and experimentally. The aim of this paper is -further theoretical study of the rough surface, but rather on a microscopical scale than on large distances. As noted in [21], the rough interface can be considered as a long-distance property and "on small scales, the interface might look quite smooth". To study this more quantitatively a new method will be presented. It will enable to calculate some basic local parameters of the disordered surface in a wide range of temperatures.

In Sec. 2 of this paper, the discrete Gaussian model [6] will be applied for the crystal surface, which is assumed to be flat (no terraces) at zero temperature. On the basis of general method [22] the exact statistical identities for DG Hamiltonian will be derived. Then, an integral operator technique will be adopted, which enables the autocorrelation function to be calculated, as well as other neighbouring correlations. As a result, the surface specific heat will be calculated in the frame of this method. In Sec. 3, the numerical results, as well as their discussion, will be presented. 


\section{Theory}

\subsection{General formulation}

The discrete Gaussian Hamiltonian is of the form [6]:

$$
\mathcal{H}=\frac{1}{2} \sum_{(i, j)} J_{i j}\left(h_{i}-h_{j}\right)^{2}
$$

where $h_{i}=0, \pm 1, \pm 2, \ldots$ represents the height of the $i$-th atomic column, and the summation over $\langle i, j\rangle$ concerns all nearest neighbours (n.n.). We assume that $J_{i j}=J$ for n.n., and we separate the Hamiltonian into two parts

$$
\mathcal{H}=\mathcal{H}_{\mathrm{cl}}+\mathcal{H}^{\prime}
$$

where

$$
\mathcal{H}_{\mathrm{cl}}=J \sum_{j \in i}^{z}\left(h_{i}-h_{j}\right)^{2}
$$

is the smallest cluster Hamiltonian. In Eq. (3), by $z$ we denote a number of lateral columns (n.n.) interacting with the central $i$-th column.

For any classical cluster Hamiltonian, $\mathcal{H}_{\mathrm{cl}}$, we can make use of the general statistical identity of the form [22]:

$$
\left\langle\{f\} f_{\mathrm{cl}}\right\rangle=\left\langle\{f\} \frac{\operatorname{Tr}_{\mathrm{cl}}\left(f_{\mathrm{cl}} \mathrm{e}^{-\beta \mathcal{H}_{\mathrm{cl}}}\right)}{\operatorname{Tr}_{\mathrm{cl}}\left(\mathrm{e}^{-\beta \mathcal{H}_{\mathrm{cl}}}\right)}\right\rangle,
$$

which is exact. By $f_{\mathrm{cl}}$ we denote any function of statistical variables from inside the cluster, and $\{f\}$ is an arbitrary function defined outside it. In particular, we can assume

$$
\{f\}=1 \text { and } \int_{\mathrm{cl}}=\left(h_{i}\right)^{k}, \quad k=1,2, \ldots
$$

Then, from Eq. (4) we obtain the following identity:

$$
\left\langle\left(h_{i}\right)^{k}\right\rangle=\left\langle\frac{\operatorname{Tr}_{\mathrm{cl}}\left\{\left(h_{i}\right)^{k} \exp \left[-\beta J \sum_{j \in i}^{z}\left(h_{i}-h_{j}\right)^{2}\right]\right\}}{\operatorname{Tr}_{\mathrm{cl}}\left\{\exp \left[-\beta J \sum_{j \in i}^{z}\left(h_{i}-h_{j}\right)^{2}\right]\right\}}\right\rangle .
$$

Substituting $k=1,2$ in Eq. (6) we have the corresponding identities for the first two moments

$$
\left\langle h_{i}\right\rangle=\left\langle\frac{\sum_{n=0}^{\infty} n \mathrm{e}^{-\beta J z n^{2}} \sinh \left(2 \beta J n \sum_{j \in i}^{z} h_{j}\right)}{\sum_{n=0}^{\infty} \mathrm{e}^{-\beta J z n^{2}} \cosh \left(2 \beta J n \sum_{j \in i}^{z} h_{j}\right)-\frac{1}{2}}\right\rangle
$$

and

$$
\left\langle\left(h_{i}\right)^{2}\right\rangle=\left\langle\frac{\sum_{n=0}^{\infty} n^{2} \mathrm{e}^{-\beta J z n^{2}} \cosh \left(2 \beta J n \sum_{j \in i}^{z} h_{j}\right)}{\sum_{n=0}^{\infty} \mathrm{e}^{-\beta J z n^{2}} \cosh \left(2 \beta J n \sum_{j \in i}^{z} h_{j}\right)-\frac{1}{2}}\right\rangle .
$$

One has to point out that identities (7) and (8) are exact within DG model. 
To calculate the statistical average on the right-hand side of Eqs. (7) and (8), we will make use of the integral operator method, presented in [22]. Let us denote for the simplicity

$$
\gamma_{i}=\sum_{j \in i}^{z} h_{j}
$$

Then, the mean value of an arbitrary function $F\left(\gamma_{i}\right)$ can be written as

$$
\left\langle F\left(\gamma_{i}\right)\right\rangle=\int_{-\infty}^{+\infty} \mathrm{d} \omega F(\omega)\left\langle\delta\left(\omega-\gamma_{i}\right)\right\rangle .
$$

Dirac's delta function is given by its integral representation

$$
\delta(x)=\frac{1}{2 \pi} \int_{-\infty}^{+\infty} \mathrm{d} t \mathrm{e}^{-\mathrm{i} t x} .
$$

Substituting (11) into (10) we obtain

$$
\left\langle F\left(\gamma_{i}\right)\right\rangle=\int_{-\infty}^{+\infty} \mathrm{d} \omega F(\omega) \frac{1}{2 \pi} \int_{-\infty}^{+\infty} \mathrm{d} t \mathrm{e}^{-\mathrm{i} \omega t}\left\langle\mathrm{e}^{\mathrm{i} t \gamma_{i}}\right\rangle .
$$

From Eq. (12) we see that the problem of the statistical a verage calculations of any function $F\left(\gamma_{i}\right)$ can be reduced to the evaluation of the expression

$$
\left\langle\mathrm{e}^{\mathrm{i} t \gamma_{i}}\right\rangle=\left\langle\exp \left(\mathrm{i} t \sum_{j \in i}^{z} h_{j}\right)\right\rangle=\left\langle\prod_{j \in i}^{z} \mathrm{e}^{\mathrm{i} t h_{j}}\right\rangle,
$$

where $h_{j}$ are the nearest neighbours of the $i$-th column.

All formulae written until now are exact. Now, in the first approximation, we will assume a decoupling, according to which the $j$-th columns, belonging to the first coordination sphere, are uncorrelated with each other, i.e. we have

$$
\left\langle\prod_{j \in i}^{z} \mathrm{e}^{\mathrm{i} t h_{j}}\right\rangle \approx\left\langle\mathrm{e}^{\mathrm{i} t h_{j}}\right\rangle^{z}
$$

In this approximation, from Eq. (12), the statistical average of any function $F\left(\gamma_{i}\right)$ can be written as

$$
\left\langle F\left(\gamma_{i}\right)\right\rangle \approx \int_{-\infty}^{+\infty} \mathrm{d} \omega F(\omega) \frac{1}{2 \pi} \int_{-\infty}^{+\infty} \mathrm{d} t \mathrm{e}^{-\mathrm{i} t(\omega-z(h))}\left\langle\mathrm{e}^{\mathrm{i} t\left(h_{j}-\langle h\rangle\right)}\right\rangle^{z},
$$

where $\langle h\rangle \stackrel{\text { df }}{=}\left\langle h_{i}\right\rangle=\left\langle h_{j}\right\rangle$.

Formula (15) is general, and can be applied for the right-hand side of identities (7) and (8). In the following, we will make use of the expansion

$$
\left\langle\mathrm{e}^{\mathrm{i} t\left(h_{j}-(h\rangle\right)}\right\rangle=\sum_{k=0}^{\infty} \frac{(\mathrm{i} t)^{k}}{k !}\left\langle\left(h_{j}-\langle h\rangle\right)^{k}\right\rangle .
$$

It is seen from Eq. (16) that central moments of the $k$-th order have to be taken into account. This can be done by the appropriate decoupling procedure which will be described below. 


\subsection{Molecular field approximalion (MFA)}

In this simplest approximation, we assume that all central moments are equal to zero, namely

$$
\left\langle\left(h_{j}-\langle h\rangle\right)^{k}\right\rangle \approx 0, \quad k=1,2, \ldots
$$

and from (16) we obtain

$$
\left\langle\mathrm{e}^{\mathrm{i} t\left(h_{j}-\langle h)\right)}\right\rangle \approx 1 .
$$

Then, Eq. (15) can be written in the form

$$
\left\langle F\left(\gamma_{i}\right)\right\rangle \stackrel{\text { MFA }}{\approx} F(z\langle h\rangle) \text {. }
$$

With the help of Eq. (19), the basic identities (7) and (8) can be written in MFA as

$$
\begin{gathered}
\langle h\rangle \approx \frac{\sum_{n=0}^{\infty} n \mathrm{e}^{-\beta J z n^{2}} \sinh (2 \beta J z n\langle h\rangle)}{\sum_{n=0}^{\infty} \mathrm{e}^{-\beta J z n^{2}} \cosh (2 \beta J z n\langle h\rangle)-\frac{1}{2}}, \\
\left\langle h^{2}\right\rangle \approx \frac{\sum_{n=0}^{\infty} n^{2} \mathrm{e}^{-\beta J z n^{2}} \cosh (2 \beta J z n\langle h\rangle)}{\sum_{n=0}^{\infty} \mathrm{e}^{-\beta J z n^{2}} \cosh (2 \beta J z n\langle h\rangle)-\frac{1}{2}},
\end{gathered}
$$

where $\left\langle h^{2}\right\rangle \stackrel{\mathrm{df}}{=}\left\langle\left(h_{i}\right)^{2}\right\rangle$.

\subsection{Gaussian approximation}

In approximation better than MFA, the central moments are given by

$$
\left\langle\left(h_{j}-\langle h\rangle\right)^{2 m+1}\right\rangle \approx 0, \quad m=0,1,2, \ldots
$$

and

$$
\begin{aligned}
& \left\langle\left(h_{j}-\langle h\rangle\right)^{2 m}\right\rangle \approx 1 \cdot 3 \cdot \ldots \cdot(2 m-1) \sigma^{2 m}=(2 m-1) ! ! \sigma^{2 m} \\
& m=0,1,2, \ldots
\end{aligned}
$$

where

$$
\sigma^{2} \stackrel{\mathrm{df}}{=}\left\langle\left(h_{j}-\langle h\rangle\right)^{2}\right\rangle .
$$

Making use of Eqs. (21) and (22) the summation in Eq. (16) can be performed, and we obtain

$$
\left\langle\mathrm{e}^{\mathrm{i} t\left(h_{j}-\langle h)\right)}\right\rangle \approx \mathrm{e}^{-t^{2} \sigma^{2} / 2}
$$

Equation (24) can be compared with the corresponding Eq. (18) obtained in MFA. We see from (24) that when the standard deviation approaches zero $(\sigma \rightarrow 0)$, then the Gaussian approximation reduces to MFA. Substituting Eq. (24) into (15) we obtain

$$
\left\langle F\left(\gamma_{i}\right)\right\rangle \approx \int_{-\infty}^{+\infty} \mathrm{d} \omega F(\omega) \frac{1}{2 \pi} \int_{-\infty}^{+\infty} \mathrm{d} t \mathrm{e}^{-\mathrm{i} t(\omega-z(h))} \mathrm{e}^{-z t^{2} \sigma^{2} / 2} .
$$

The integration over $t$-parameter in Eq. (25) can be performed analytically, since we make use of the mathematical formula

$$
\int_{-\infty}^{+\infty} \mathrm{d} x \mathrm{e}^{ \pm \mathrm{i} p x} \mathrm{e}^{-q^{2} x^{2}}=\frac{\sqrt{\pi}}{q} \mathrm{e}^{-p^{2} / 4 q^{2}}
$$


Then, from Eqs.(26) and (25) we will obtain

$$
\left\langle F\left(\gamma_{i}\right)\right\rangle \approx \int_{-\infty}^{+\infty} \mathrm{d} \omega F(\omega) \frac{1}{\sqrt{2 \pi z} \sigma} \mathrm{e}^{-(\omega-z(h))^{2} / 2 z \sigma^{2}} .
$$

Formula (27) can be then used for the evaluation of right-hand sides of Eqs. (7) and $(8)$ in Gaussian approximation. Finally, we obtain

$$
\begin{aligned}
& \langle h\rangle \approx \frac{1}{\sqrt{2 \pi} \sigma} \int_{-\infty}^{+\infty} \mathrm{d} \omega \mathrm{e}^{-\omega^{2} / 2 \sigma^{2}} \frac{\sum_{n=-\infty}^{+\infty} n \exp \left[-\beta J z\left(n-\frac{\omega}{\sqrt{z}}-\langle h\rangle\right)^{2}\right]}{\sum_{n=-\infty}^{+\infty} \exp \left[-\beta J z\left(n-\frac{\omega}{\sqrt{z}}-\langle h\rangle\right)^{2}\right]}, \\
& \left\langle h^{2}\right\rangle \approx \frac{1}{\sqrt{2 \pi} \sigma} \int_{-\infty}^{+\infty} \mathrm{d} \omega \mathrm{e}^{-\omega^{2} / 2 \sigma^{2}} \frac{\sum_{n=-\infty}^{+\infty} n^{2} \exp \left[-\beta J z\left(n-\frac{\omega}{\sqrt{z}}-\langle h\rangle\right)^{2}\right]}{\sum_{n=-\infty}^{+\infty} \exp \left[-\beta J z\left(n-\frac{\omega}{\sqrt{z}}-\langle h\rangle\right)^{2}\right]},
\end{aligned}
$$

where

$$
\sigma^{2}=\left\langle h^{2}\right\rangle-\langle h\rangle^{2} .
$$

It is seen that (28) and (29) are two coupled equations which have to be solved numcrically. In the above equations the self-consistency conditions $\langle h\rangle=\left\langle h_{i}\right\rangle=$ $\left\langle h_{j}\right\rangle$ and $\left\langle h^{2}\right\rangle=\left\langle\left(h_{i}\right)^{2}\right\rangle=\left\langle\left(h_{j}\right)^{2}\right\rangle$ have been assumed.

\subsection{The n.n. correlation function}

Since we are interested in obtaining the formula for n.n. correlation function, we shall return to the general identity (4). Substituting $\{f\}=h_{j}(j \in i)$ and $f_{\mathrm{cl}}=h_{i}$ we obtain $\left\langle h_{i} h_{j}\right\rangle=\left\langle h_{j} F\left(\gamma_{i}\right)\right\rangle$, and we can basically follow the whole route described in this section. However, now the new autocorrelation function will occur in the neighbourhood of the $i$-th lattice site. Namely, analogously to Eq. (14) we can write

$$
\begin{aligned}
& \left\langle h_{j} \prod_{k=1}^{z} \mathrm{e}^{\mathrm{i} t h_{k}}\right\rangle \approx\left\langle h_{j} \mathrm{e}^{\mathrm{i} t h_{j}}\right\rangle \cdot\left\langle\mathrm{e}^{\mathrm{i} h t_{k}}\right\rangle^{(z-1)} \\
& =\mathrm{e}^{\mathrm{i} t z\langle h\rangle}\left\langle h_{j} \mathrm{e}^{\mathrm{i} t\left(h_{j}-(h)\right)}\right\rangle \cdot\left\langle\mathrm{e}^{\mathrm{i} t\left(h_{k}-\langle h\rangle\right)}\right\rangle^{(z-1)}
\end{aligned}
$$

and we find in Gaussian approximation

$$
\left\langle h_{j} \mathrm{e}^{\mathrm{j} t\left(h_{j}-(h)\right)}\right\rangle \approx \mathrm{e}^{-t^{2} \sigma^{2} / 2}\left(\mathrm{i} t \sigma^{2}+\langle h\rangle\right) .
$$

Making use of the relations (32) and (24), Eq. (31) can be presented as

$$
\begin{aligned}
& \left\langle h_{j} \prod_{k=1}^{z} \mathrm{e}^{\mathrm{i} t h_{k}}\right\rangle \approx \mathrm{e}^{\mathrm{i} t z}\langle h\rangle \mathrm{e}^{-z t^{2} \sigma^{2} / 2}\left(\mathrm{i} t \sigma^{2}+\langle h\rangle\right) \\
& =\mathrm{e}^{-z t^{2} \sigma^{2} / 2}\left(\langle h\rangle+\frac{\sigma^{2}}{z} \frac{\partial}{\partial\langle h\rangle}\right) \mathrm{e}^{\mathrm{i} t z\langle h\rangle} .
\end{aligned}
$$

Now, Eq. (33) enables us to calculate $\left\langle h_{j} F\left(\gamma_{i}\right)\right\rangle$ (again making use of the formula (26)). We find

$$
\left\langle h_{j} F\left(\gamma_{i}\right)\right\rangle \approx \int_{-\infty}^{+\infty} \mathrm{d} \omega F(\omega) \frac{1}{\sqrt{2 \pi z} \sigma} \frac{\omega}{z} \mathrm{e}^{-(\omega-z\langle h))^{2} / 2 z \sigma^{2}} .
$$


Finally, for the n.n. correlation function we obtain in Gaussian approximation

$$
\begin{aligned}
\left\langle h_{i} h_{j}\right\rangle & \approx \frac{1}{\sqrt{2 \pi} \sigma} \int_{-\infty}^{+\infty} \mathrm{d} \omega \frac{\omega}{\sqrt{z}} \mathrm{e}^{-\omega^{2} / 2 \sigma^{2}} \\
\times & \frac{\sum_{n=-\infty}^{+\infty} n \exp \left[-\beta J z\left(n-\frac{\omega}{\sqrt{z}}-\langle h\rangle\right)^{2}\right]}{\sum_{n=-\infty}^{+\infty} \exp \left[-\beta J z\left(n-\frac{\omega}{\sqrt{z}}-\langle h\rangle\right)^{2}\right]}+\langle h\rangle^{2},
\end{aligned}
$$

wherc $\langle h\rangle$ and $\sigma$ are given by Eqs. (28)-(30).

\subsection{The surface specific heat}

The surface contribution to the specific heat can be calculated from the temperature dependence of the surface energy. Recalling the Hamiltonian (1), we have

$$
E=\langle\mathcal{H}\rangle=\frac{1}{2} \sum_{(i, j)} J_{i j}\left\langle\left(h_{i}-h_{j}\right)^{2}\right\rangle=\frac{N z J}{2}\left\langle\left(h_{i}-h_{j}\right)^{2}\right\rangle
$$

and the mean energy per one column is then given by

$$
\frac{E}{N}=z J\left(\left\langle h^{2}\right\rangle-\left\langle h_{i} h_{j}\right\rangle\right),
$$

where $N$ is a total number of atomic columns, forming the surface. The specific heat per one column is then define as

$$
\frac{C}{N}=\frac{1}{N} \frac{\partial E}{\partial T}=z J \frac{\partial}{\partial T}\left(\left\langle h^{2}\right\rangle-\left\langle h_{i} h_{j}\right\rangle\right) .
$$

Because both $\left\langle h^{2}\right\rangle$ and $\left\langle h_{i} h_{j}\right\rangle$ functions are calculated vs. dimensionless variable $k T / J z$, it is convenient to denote it by

$$
\alpha=\frac{k T}{J z}
$$

and instead of Eq. (37) we will have the formula

$$
\frac{C}{k N}=\frac{\partial}{\partial \alpha}\left(\left\langle h^{2}\right\rangle-\left\langle h_{i} h_{j}\right\rangle\right) \text {. }
$$

Equation (39) will be then used for the numerical calculations of the surface specific heat, once we have calculated the autocorrelation $\left\langle h^{2}\right\rangle$ and n.n. correlation function $\left\langle h_{i} h_{j}\right\rangle$ (see Eqs. (28)-(30) and (35)).

\subsection{High-temperature approximation}

Equations (28)-(30), (35) and (39), obtained in Gaussian approximation, can be solved numerically for any temperature. However, for $k T / J z \geq 1$ we can substitute the DG model by a continuous one, and the summations in Eqs. (28)-(30) and (35) can be then replaced by the integration

$$
\sum_{n=-\infty}^{+\infty} \longrightarrow \int_{-\infty}^{+\infty} \mathrm{d} n \text {. }
$$


In this approximation, the integrals over $\mathrm{d} n$, as well as over $\mathrm{d} \omega$, can be easily performed analytically. Further, we will assume that the total number of atoms is constant (i.e. no evaporation or deposition process takes place) and we obtain from Eqs. (28)-(30), with the help of (40):

$$
\langle h\rangle=0
$$

and

$$
\left\langle h^{2}\right\rangle=\sigma^{2}=\frac{k T}{J} \frac{1}{2(z-1)}
$$

for Gaussian approximation. Correspondingly, for MFA one obtains from Eq. (20):

$$
\left\langle h^{2}\right\rangle \stackrel{\mathrm{MFA}}{\approx} \frac{1}{2} \frac{k T}{J z} .
$$

For the n.n. correlation function we will have the high-temperature approximation of Eq. (35) in the form

$$
\left\langle h_{i} h_{j}\right\rangle=\frac{\sigma^{2}}{z}=\frac{k T}{J z} \frac{1}{2(z-1)} .
$$

At last, for the surface specific heat, from Eqs. (39), (42) and (44) we find

$$
\frac{C}{k N}=\frac{1}{2} \text {. }
$$

The numerical results based on the formulae derived here, will be prescnted in the next section.

\section{The numerical results and discussion}

We will consider the simplest physical situation, when the total number of atoms is constant, and the thermally induced roughness is only due to the stochastic displacement of atoms, perpendicular to the crystal surface. Since this displacement has the same probability up and down, the mean column height remains also constant. One of the solutions is $\langle h\rangle=0$, which satisfies Eqs. (19) for MFA and (28) for Gaussian approximation. We will assume that $\langle h\rangle=0$ defines the position of thermally unperturbed surface for very low temperatures. With the above assumptions made, the mean square of the column height, $\left\langle h^{2}\right\rangle$, is equal to $\sigma^{2}$, and can be considered as a roughening parameter for the surface.

In Fig. 1 the $\left\langle h^{2}\right\rangle$ function, calculated from Eq. (20) in MFA, is presented vs. temperature. One can see that using the dimensionless variable $\alpha=k T / J z$, the shape of the curve is not dependent on the $z$-number, i.e. on the crystal facet. The dashed line in Fig. 1 is an extrapolation of the high-temperature approximation (cp. Eq. (43)). We see that the numerical calculations based on Eq. (20) fit well the high-temperature approximation, if only $k T / J z \geq 1$. This temperature range corresponds to continuous Gaussian model and represents the behaviour of a classical oscillator. On the other hand, in the low temperature region, the DG model differs markedly from a continuous one, as is seen in Fig. 1 . It is seen also that whereas the temperature increases from $T=0$, the surface remains quite smooth until $k T / J z \approx 0.1$. 


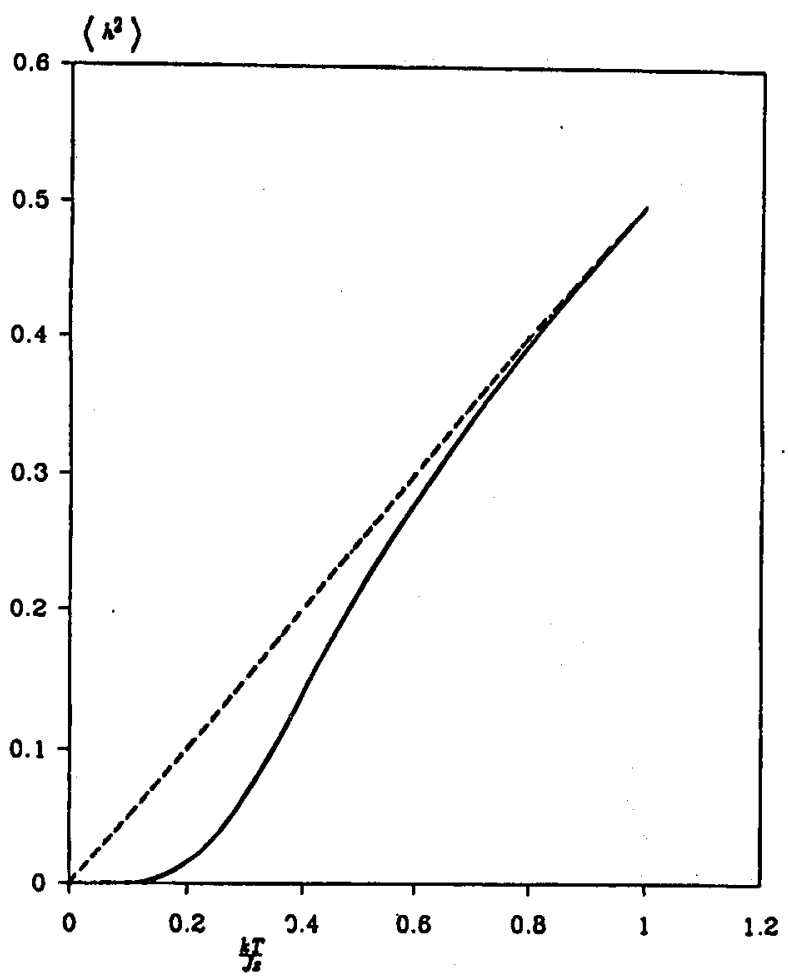

Fig. 1. The mean square fo the column height $\left\langle h^{2}\right\rangle$ vs. temperature $k T / J z$, calculated for DG model in molecular field approximation. The dashed line represents the result for a continuous Gaussian model.

In Fig. 2 the $\left\langle h^{2}\right\rangle$-parameter is calculated from Eq. (29), in Gaussian approximation. Three curves for $z=3,4$, and 6 are presented, corresponding to surface having the honeycomb, square, and triangular symmetry, respectively. In this case, although the shape of the curves is similar to that of MFA (dashed curve), we can distinguish between different crystal facets. All these curves lie above the MFA result, and the smallest $z$-number corresponds to the highest values of $\left\langle h^{2}\right\rangle$. This illustrates that the surface with smaller coordination number can become rough more easily than the surface with higher $z$, which is more stable. The greatest differences in values for these curves are in high-temperature region, $\alpha \geq 1$, which can be predicted well by Eq. (42).

We can conclude from Fig. 2 that the Gaussian approximation is much more accurate than MFA, since autocorrelations of the atomic columns are then taken into account more properly. However, for very small temperatures, both approximations are of the same validity, because all correlations are then negligible. 


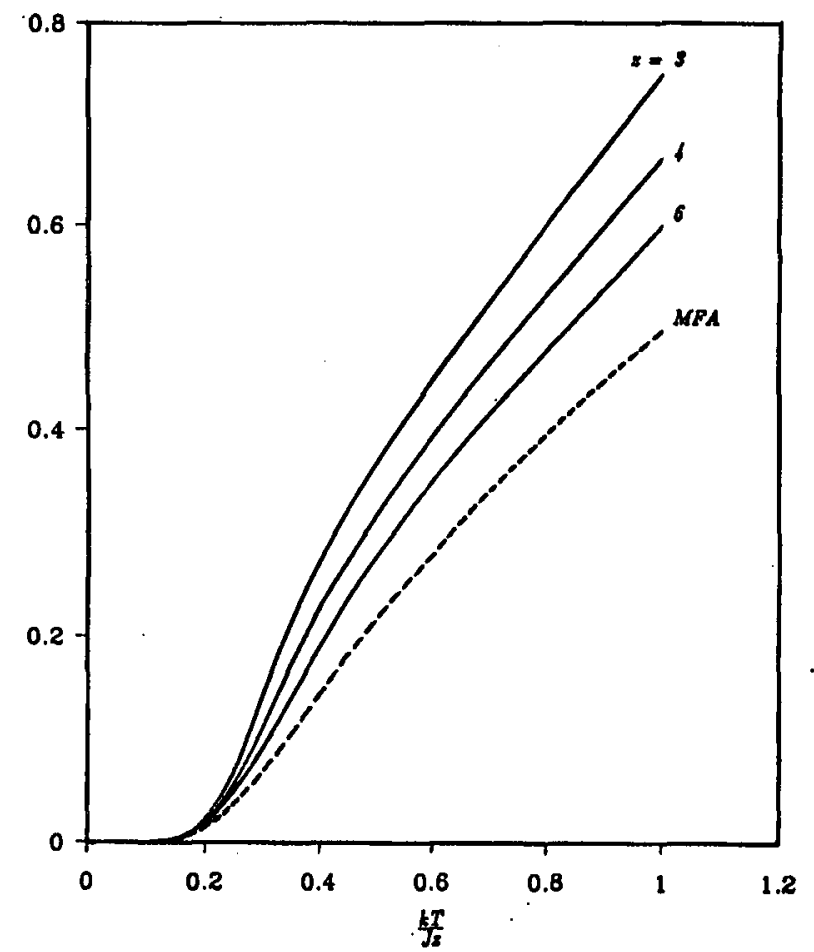

Fig. 2. The mean square of the column height $\left\langle h^{2}\right\rangle$ vs. temperature $k T / J z$, calculated for DG model in Gaussian approximation for correlations. The number of nearest ncighbours $z=3,4$ and 6 correspond to honeycomb, square, and triangular lattice, respectively. The dashed line shows the MFA result as in Fig. 1.

In Fig. 3 the n.n. correlation functions $\left\langle h_{i} h_{j}\right\rangle$ are presented vs. $\alpha$, for the same $z$-numbers as in Fig. 2. The curves were calculated in Gaussian approximation, from Eq. (35). Comparing Figs. 3 and 2 we see that the values of $\left\langle h_{i} h_{j}\right\rangle$ are much smaller than corresponding autocorrelations. One can note that in high-temperature region the magnitude of n.n. correlations is reduced by factor $z$ in comparison with $\left\langle h^{2}\right\rangle$ (see Eq. (44)). Moreover, for different $z$, the correlations are relatively more spread than autocorrelations. Both $\left\langle h_{i} h_{j}\right\rangle$ and $\left\langle h^{2}\right\rangle$ are next used for the specific heat calculations.

The surface specific heat per one lattice site, in Boltzmann constant units, is presented in Fig. 4. Three lattices with $z=3,4$, and 6 are examined. We found that the specific heat has a finitc maximum at $k T / J z=0.265,0.286$, and 0.312 for these three lattices, respectively. One can note that this maximum becomes flatten when $z$-number is increased. For high temperatures the specific heat is constant and becomes independent on structure (cp. Eq. (45)). This fact again reminds the 


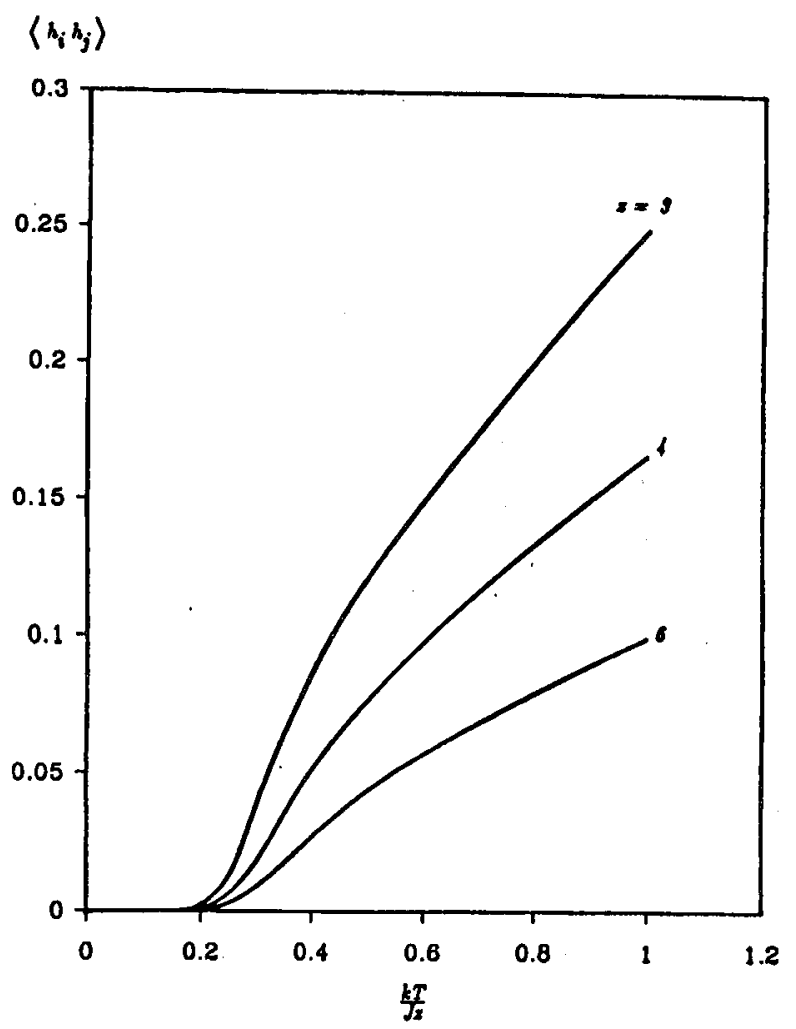

Fig. 3. The nearest neighbour correlation function $\left\langle h_{i} h_{j}\right\rangle$, calculated for DG model vs. temperature $k T / J z$, for the lattices with $z=3,4$, and 6 .

property of a classical oscillator. On the other hand, for $T \rightarrow 0$, the vanishing of the specific heat is in agreement with the principles of thermodynamics.

The presented numerical results concern the properties of the rough surface on a microscale. Using our method we did not find any singularity in $\left\langle h^{2}\right\rangle$ dependence on temperature. However, it is possible that for the surface which is terraced in the ground state, a long-distant pair-correlation function will diverge at some temperatures. Therefore, our result does not exclude the phase transition of the Kosterlitz-Thouless type for vicinity surfaces. The illustration for such situation is given in [8], where the results of MC simulations are compared for (001) and vicinal surfaces.

The experimental study of alomic roughening of Ge (001) surface, performed with X-ray diffraction [23], give a support for the validity of our model. In the paper [23], the simple three level $(+1,0,-1)$ SOS model has been used to explain satisfactorily the observed creation of adatoms and vacancies on that surface.

In another work [24] the continuous Gaussian model (spring model) has been adopted for studying the surface roughness of ternary alloys. The results obtained 


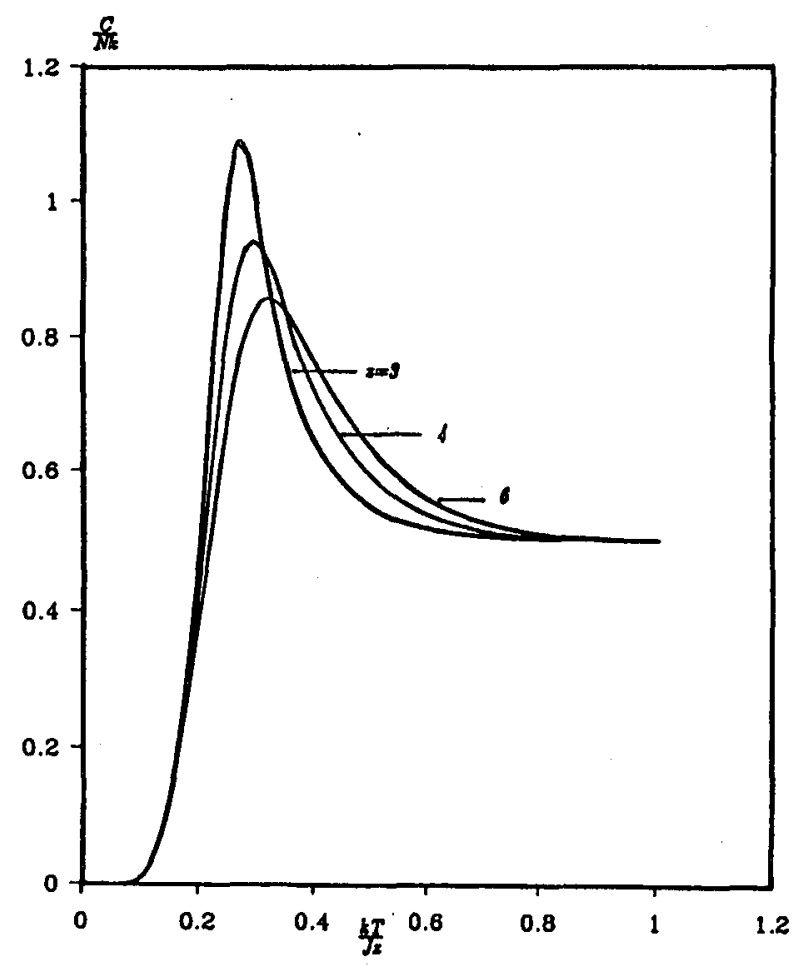

Fig. 4. The roughening specific heat $C / N k$ per one lattice site (in Boltzmann constant unit), calculated for DG model as a function of temperature $k T / J z$, for the lattices with $z=3,4$, and 6 .

in [24] by numerical simulations are in qualitalive agreement with the predictions of the present paper and can serve as a good illustration for our model at high temperatures.

In conclusion, we see that the presented method of the surface roughness description can be adopted for various crystal surfaces in the whole temperature region. Using the simple DG model we can investigate the basic statistical properties of the rough surface which, in turn, can be related to the experimental situation. Although the model considered in this paper is very simple and it serves here as an illustration of the method rather than for the description of any particular material, it can be easily extended for some more complex physical situations.

The first improvement on the model can be done after introducing the quadratic term into the Hamiltonian, which originates from the crystalline anisotropy. As we know, the presence of the anisotropy is essential for the surface premelting phenomena. 
Further improvement may be achieved when the surface disorder is taken into account, via possible fluctuations of intercolumnar interaction $J_{i j}$. Those fluctuations play an important role when some impurities are present at the surface.

At last, the surface dynamics can be also introduced into the theory. Using the concept of chemical potential, we could investigate the surface growth at different temperatures and under external pressure.

We are convinced that the method outlined in this work will be also useful for studying the solid-liquid interfaces as well as the roughening of ultra-thin films.

It is obvious that the accuracy of calculations, in the frame of this method, depends on the correlation decoupling scheme. The possible way to improve this procedure would be the method of Matsudaira [25] known in magnetism, since then the successive pair correlations are taken into account. Those correlations, in turn, can be determined from the series of exact identities, generated by the formula (4).

The above mentioned problems will be studied in the forthcoming papers.

\section{References}

[1] W.K. Burton, N. Cabrera, Disc. Faraday Soc. 5, 40 (1949).

[2] W.K. Burton, N. Cabrera, F.C. Franck, Philos. Trans. R. Soc. A 243, 299 (1951).

[3] P. Bennema, G.H. Gilmer, in: Crystal Growth, an Inlroduction, Ed. P. Hartman, North IIolland, Amsterdam 1973, p. 63.

[4] J.D. Weeks, G.H. Gilmer, H.J. Leamy, Phys. Rev. Lelt. 31, 549 (1973).

[5] S.T. Chui, J.D. Weeks, Phys. Rev. B 14, 4978 (1976).

[6] J.D. Weeks, in: Ordering in Strongly Fluctuating Condensed Malter Systems, Ed. T. Riste, Plenum Press, New York 1980, p. 293.

[7] M. Wortis, in: Ordering in Two Dimensions, Ed. S.K. Sinha, Elsevier North-Holland, Amsterdam 1980, p. 3.

[8] H. van Beijeren, I. Nolden, in: Structure and Dynamics of Surfaces II, Eds. W. Schommers, P. von Blackenhagen, Springer-Verlag, Berlin 1987, p. 259.

[9] D. Nenov, A. Trayanov, Surf. Sci. 213, 488 (1989).

[10] P. Bennema, in: Kinetics of Ordering and Growth at Surfaces, Ed. Max G. Lagally, Plenum Press, New York 1990, p. 415.

[11] J.M. Kosterlitz, J. Phys. C 7, 1046 (1974).

[12] J.E. Avron, L.S. Balfour, C.G. Kuper, L. Landau, S.G. Lipson, L.S. Schulman, Phys. Rev. Lell. 45, 814 (1980).

[13] K.S. Liang, E.B. Sirota, K.L. D'Amico, G.J. Hughes, S.K. Sinha, W.N. Unertl, in: The Structure of Surfaces II, Eds. J.F. van der Veen, M.A. Van Hove, Springer-Verlag, Berlin 1988, p. 509.

[14] E.H. Conrad, L.R. Allen, D.L. Blanchard, T. Engel, in Ref. [13], p. 514.

[15] F. Fabre, B. Salanon, J. Japujoulade, in Ref. [13], p. 520.

[16] J. Japujoulade, B. Salanon, F. Fabre, in Ref. [10], p. 355.

[17] B. Mutaftschiev, in Ref. [10], p. 169.

[18] D.D. Vvedensky, S. Clarke, K.J. Hugill, A.K. Myers-Beaghton, M.R. Wilby, in Ref. [10], p. 297. 
[19] H.P. Bonzel, U. Breuer, Acta Phys. Pol. A 81, 265 (1992).

[20] O.G. Mouritsen, in Ref. [10], p. 1.

[21] M. Schick, in: Introduction to Wetting Phenomena, Course 9, Les Houches, Session XLVIII, Eds. J. Charvolin, J.F. Joanny, J. Zinn-Justin, Elsevier Sci. Publ. B.V., Amsterdam 1990, p. 464.

[22] T. Balcerzak, J. Magn. Magn. Mater. 97, 152 (1991).

[23] A.D. Johnson, C. Norris, J.W.M. Frenken, H.S. Derbyshire, J.E. MacDonald, R.G. Van Silfhout, J.M. van der Veen, Phys. Rev. B 44, 1134 (1991).

[24] M.F. Thorpe, W. Jin, S.D. Mahanti, in: Disorder in Condensed Malter Physics, Eds. J.A. Blackman, J. Tagüeña, Clarendon Press, Oxford 1991, p. 24.

[25] N. Matsudaira, J. Phys. Soc. Jpn. 35, 1593 (1973). 